\title{
Enzastaurin Hydrochloride
}

National Cancer Institute

\section{Source}

National Cancer Institute. Enzastaurin Hydrochloride. NCI Thesaurus. Code C26658.

The hydrochloride salt of enzastaurin, a synthetic macrocyclic bisindolemaleimide with potential antineoplastic activity. Binding to the ATP-binding site, enzastaurin selectively inhibits protein kinase $\mathrm{C}$ beta, an enzyme involved in the induction of vascular endothelial growth factor (VEGF)-stimulated neo-angiogenesis. This agent may decrease tumor blood supply and so tumor burden. 